Caroline Soares Cristofari Emer ${ }^{1}$

Julo Alejandro Peña Duque ${ }^{1}$

Ana Lúcia Lettı Müller ${ }^{2}$

RejANE Gus $^{3}$

Maria Teresa Vielra Sanseverino ${ }^{4}$

ANDRÉ ANJOS DA SILVA ${ }^{1}$

José ANtONIO de AzEVEDo Magalhães ${ }^{5}$

Artigo Original

Palavras-chave

Feto/anormalidades

Ultrassonografia pré-natal

Aberrações cromossômicas

Trissomia

Aneuploidia

Keywords

Fetus/abnormalities Ultrasonography prenatal Chromosome aberrations

Trisomy

Aneuploidy

\title{
Prevalência das malformações congênitas identificadas em fetos com trissomia dos cromossomos 13, 18 e 21
}

\author{
Prevalence of congenital abnormalities identified in \\ fetuses with 13, 18 and 21 chromosomal trisomy
}

\section{Resumo}

OBJETIVO: Descrever a prevalência das malformações encontradas nos fetos com trissomia dos cromossomos 13 , 18 e 21, identificando as mais frequentes em cada condição. MÉTODOS: Estudo transversal retrospectivo, com análise dos casos de trissomias dos cromossomos 13, 18 e 21 que foram diagnosticados pelo cariótipo fetal obtido por amniocentese/cordocentese, entre outubro de 1994 e maio de 2014, em um Hospital de Ensino da região Sul do Brasil. Foram descritas as malformações identificadas no exame ultrassonográfico morfológico e, posteriormente, confirmadas em exames do recém-nascido e/ou por necropsia fetal. Os resultados foram analisados por meio do teste de Fisher e da análise de variância (ANOVA). O nível de significância empregado foi 5\% ( $p=0,05)$. RESULTADOS: Em 840 exames realizados, foram diagnosticados 69 casos de trissomias; nove deles foram excluídos por desfecho ocorrido fora do Hospital de Clínicas de Porto Alegre ou prontuário incompleto, restando 60 casos (nove de trissomia do cromossomo 13, 26 do cromossomo 18 e 25 do cromossomo 21). As cardiopatias ocorreram, na maioria dos casos, nos três grupos; a comunicação interventricular foi mais prevalente, em 66,7\% do grupo da trissomia 13 . As anomalias gastrintestinais aconteceram mais no grupo da trissomia 18, principalmente a onfalocele $(38,5 \% ; p<0,01)$. As anomalias geniturinárias foram significativamente mais frequentes no grupo da trissomia 13 (pielectasia com 55,6\%$\mathrm{p}<0,01$; genitália ambígua com 33,3\% - p=0,011. Defeitos do sistema nervoso central foram identificados em todos os casos de trissomia 13. Fendas faciais foram mais prevalentes dentre os fetos com trissomia $13(66,7 \% ; p<0,01)$. Malformações nas mãos e nos pés tiveram diferenças estatísticas entre os grupos de trissomia. Os defeitos nas mãos ocorreram em 50\% dos casos de trissomia 18 e em 44,4\% dos casos de 13 ( $p<0,01$ ); pé torto congênito foi mais comum no grupo da trissomia 18, descrito em $46,2 \%$ dos fetos $(p<0,01)$. As malformações foram identificadas em 50,9; 27,3 e 21,7\% dos casos de trissomias 18, 13 e 21, respectivamente. CONCLUSÃO: Muitas malformações identificadas na ultrassonografia são sugestivas de trissomias e mostram-se ferramentas importantes para o diagnóstico etiológico e aconselhamento genético pré-natal e pré-concepcional.

\section{Abstract}

PURPOSE: To describe the prevalence of malformations found in fetuses with trisomy of chromosomes 13, 18 and 21 by identifying the most frequent within each condition. METHODS: A retrospective cross-sectional study with the analysis of trisomy cases of chromosomes 13, 18 and 21 diagnosed through fetal karyotype obtained by amniocentesis/ cordocentesis, between October 1994 and May 2014, at a Teaching Hospital in Brazil Southern Region. Malformations identified through morphological ultrasonography were described and, subsequently, confirmed in newborn examinations and/or fetal autopsy. The results were analyzed using Fisher's test and analysis of variance (ANOVA), with a 5\% level of significance ( $p=0.05$ ). RESULTS: Sixty-nine cases of trisomy were diagnosed among 840 exams; nine were excluded due to outcome outside Hospital de Clínicas de Porto Alegre or incomplete records, remaining 60 cases (nine cases of chromosome 13 trisomy, 26 of chromosome 18, and 25 of chromosome 21). In all three groups, heart disease occurred in most cases; the ventricular septal defect was more prevalent and occurred in $66.7 \%$ of the trisomy 13

Correspondência

Caroline Soares Cristofori Emer Serviço de Ginecologia e Obstetricia do Hospital de Clinicas de Porto Alegre Rua Ramiro Barcelos, 2.350 - Sala 1.125

(EP: 90035 -903 Porto Alegre (RS), Brasil

Recebido

$30 / 04 / 2015$

Aceito com modificarộes

$16 / 06 / 2015$
Setor de Medicina Fetal do Serviço de Ginecologia e Obstetrícia do Hospital de Clínicas de Porto Alegre - HCPA - Porto Alegre (RS), Brasil. IPrograma de Residência Médica, Hospital de Clínicas de Porto Alegre - HCPA - Porto Alegre (RS), Brasil.

${ }^{2}$ Grupo de Medicina Fetal do Serviço de Ginecologia e Obstetrícia e Centro Obstétrico, Hospital de Clínicas de Porto Alegre - HCPA Porto Alegre (RS), Brasil.

${ }^{3}$ Laboratório de Diagnóstico Pré-Natal do Serviço de Genética Médica e Grupo de Medicina Fetal do Serviço de Ginecologia e Obstetrícia, Hospital de Clínicas de Porto Alegre - HCPA - Porto Alegre (RS), Brasil.

${ }^{4}$ Serviço de Genética e Setor de Medicina Fetal do Serviço de Ginecologia e Obstetrícia, Hospital de Clínicas de Porto Alegre - HCPA Porto Alegre (RS), Brasil.

${ }^{5}$ Departamento de Ginecologia e Obstetrícia, Faculdade de Medicina, Universidade Federal do Rio Grande do SUl - UFRGS - Porto Alegre (RS), Brasil; Grupo de Medicina Fetal do Serviço de Ginecologia e Obstetrícia, Hospital de Clínicas de Porto Alegre - HCPA Porto Alegre (RS), Brasil.

Conflito de interesses: não há. 
group. Gastrointestinal abnormalities were more prevalent in the trisomy 18 group, especially omphalocele (38.5\%; $<<0.01)$. Genitourinary anomalies were more significantly frequent in the trisomy 13 group (pyelectasis, $55.6 \%-p<0.01$; ambiguous genitalia, 33.3\% - p=0.01). Central nervous system defects were identified in all cases of trisomy 13. Facial cracks were significantly more prevalent among fetuses with trisomy 13 (66.7\%; $\mathrm{p}<0.01$ ). Hand and feet malformations significantly differed among the trisomy groups. Hand defects occurred in 50\% of trisomy 18 cases, and in $44.4 \%$ of all trisomy 13 cases ( $<<0.01)$; congenital clubfoot was more common in the trisomy 18 group, being detected in $46.2 \%$ of fetuses $(p<0.01)$. The abnormalities were found in 50.9, 27.3 and $21.7 \%$ of trisomy 18, 13 and 21 cases respectively. CONCLUSION: Many fetal malformations identified at ultrasound are suggestive of trisomy and represent an important tool for etiologic diagnosis and prenatal and pre-conception genetic counseling.

\section{Introdução}

As aneuploidias são as anormalidades genéticas mais comuns detectadas durante o pré-natal. Dentre as aneuploidias, as trissomias envolvendo os cromossomos 13,18 e 21 são as mais frequentemente diagnosticadas ${ }^{1}$.

As cromossomopatias ocorrem em 0,1 a $0,2 \%$ dos nascidos vivos, sendo a trissomia do cromossomo 21 a mais frequente $^{2}$. A ultrassonografia morfológica realizada entre 16 e 23 semanas de gestação possui uma taxa para detecção de trissomia do cromossomo 21 de $75 \%$, com uma taxa de falso-positivo de 10 a $15 \%{ }^{3}$. A trissomia do cromossomo 18 é a segunda trissomia autossômica identificada em nascidos vivos, sendo mais comum no sexo feminino ${ }^{4}$. Os achados ultrassonográficos desta trissomia são abundantes e o diagnóstico é relativamente fácil, com sensibilidade de 80 a $100 \%$. A terceira trissomia mais comum é a do cromossomo 13, a qual está associada a graves anomalias do sistema nervoso central (SNC), cardiopatias e malformações do trato gastrintestinal. A taxa de detecção ultrassonográfica desta trissomia é de $90 \%{ }^{6}$. As trissomias dos cromossomos $13 \mathrm{e}$ 18 são graves, com diversas malformações concomitantes, o que resulta em alta taxa de perdas fetais e neonatais.

Apesar dos inúmeros marcadores ultrassonográficos pesquisados nos estudos ao longo dos anos, muitos fetos com trissomias não demonstram achados significantes, tornando a deteç̧ão de aneuploidias ainda limitada ${ }^{6}$. Além disso, algumas malformações podem ser facilmente diagnosticadas por meio da ultrassonografia, outras precisam ser especialmente pesquisadas. Desde 1992, Nicolaides et al. ${ }^{7}$ observaram que quanto maior o número de malformações fetais identificadas, maior o risco de aneuploidias. No entanto, pode ser difícil para o obstetra distinguir se o achado ultrassonográfico fetal pode ser considerado apenas como uma variante da normalidade ou reflete um aumento do risco ${ }^{6}$. Da mesma forma, na ausência de defeitos maiores e marcadores já descritos para a trissomia do cromossomo 21 , há um risco sete vezes menor desta cromossomopatia ${ }^{8}$.

Diante disso, presume-se que os ultrassonografistas obstétricos precisam conhecer as malformações mais frequentemente associadas a cada anomalia cromossômica ${ }^{7}$. Essa realidade se aplica especialmente nas regiões em que métodos mais sofisticados de diagnóstico pré-natal ainda não são acessíveis. O diagnóstico pré-natal precoce de aneuploidias propicia à família tempo para se preparar e se informar sobre o manejo da gestação ${ }^{6}$.

O objetivo deste trabalho foi descrever as principais malformações encontradas nos fetos com trissomia dos cromossomos 13,18 e 21 no meio médico, identificando aquelas mais frequentes dentro de cada trissomia e discutindo estes dados em consonância com a literatura atual a respeito do tema.

\section{Métodos}

Foi realizado um estudo transversal retrospectivo, de outubro de 1994 até maio de 2014, com as gestantes que foram submetidas à amniocentese ou cordocentese para pesquisa de cariótipo fetal no Setor de Medicina Fetal do Serviço de Ginecologia e Obstetrícia do Hospital de Clínicas de Porto Alegre (HCPA), Rio Grande do Sul, Brasil. Foram incluídos os fetos com diagnóstico de trissomia dos cromossomos 13, 18 e 21 naquele período. Os autores assinaram o termo de responsabilidade para o uso de dados de prontuário, e a pesquisa foi aprovada pelo Comitê de Pesquisa e Ética em Saúde do grupo de pesquisa e pós-graduação do HCPA, sob o número 06-038. Foram excluídos os casos em que o desfecho da gestação não ocorreu no HCPA e aqueles cujos prontuários não continham informações sobre os dados pesquisados.

As gestantes, cujos fetos apresentaram aumento de translucência nucal (TN), malformações detectadas em ultrassonografia obstétrica ou com risco aumentado para cromossomopatias atendidas no ambulatório de pré-natal do HCPA e na rede primária referenciada, foram encaminhadas para o Setor de Medicina Fetal, no qual foram submetidas ao exame de ultrassonografia obstétrica morfológica e à coleta de material para cariótipo fetal. O diagnóstico de trissomia foi dado pelo resultado do cariótipo.

As variáveis analisadas foram: idade materna em anos, paridade (primíparas ou multíparas), idade gestacional ao diagnóstico (segundo e terceiro trimestres), sexo fetal (masculino e feminino), motivo do encaminhamento ao Setor de Medicina Fetal, malformações encontradas no exame ultrassonográfico morfológico, que, posteriormente, foram confirmadas em exames do recém-nascido e/ou necropsia fetal e desfecho da gestação (óbito intraútero ou nativivo).

Os dados foram analisados com o programa SPSS versão 22, utilizando-se média, desvio padrão e contagem 
em porcentagem para variáveis quantitativas de distribuição simétrica. As médias foram comparadas por meio de análise de variância (ANOVA) e os dados categóricos, pelo teste de Fisher. O nível de significância adotado foi de $5 \%(\mathrm{p}=0,05)$.

\section{Resultados}

Foram realizadas 840 amniocenteses/cordocenteses para pesquisa de cariótipo fetal no período do estudo. Foram diagnosticados 69 casos de trissomias, nove do cromossomo 13, 30 do cromossomo 18 e 30 do cromossomo 21. Nove casos foram excluídos (cinco casos de trissomia do cromossomo 21 e quatro casos de trissomia do cromossomo 18), em função do desfecho da gestação ter ocorrido fora do HCPA ou por terem prontuário incompleto. A Tabela 1 descreve a distribuição das trissomias segundo os dados gestacionais materno-fetais encontrados, os motivos de encaminhamento para o Setor de Medicina Fetal e o desfecho da gestação.

A média de idade materna nos três grupos de trissomia mostrou tendência a uma diferença entre os grupos, sendo possivelmente mais precoce nos casos de trissomia do cromossomo 13 (30,4 anos) e mais tardia, na trissomia do cromossomo 21 (37 anos). Observou-se ainda que duas pacientes do grupo de casos de trissomia $13(22,2 \%)$, 13 pacientes do grupo de casos de trissomia 18 (50\%) e 17 do grupo de casos de trissomia $21(68 \%)$ tinham mais de 35 anos, totalizando 32 pacientes (53,3\% dos casos).

Tabela 1. Distribuição das trissomias de acordo com os dados gestacionais materno-fetais, os motivos de encaminhamento para o Setor de Medicina Fetal e o desfecho da gestação

\begin{tabular}{|c|c|c|c|c|}
\hline Características (\%) & $\begin{array}{c}\text { Trissomia } 13 \\
(\mathrm{n}=9)\end{array}$ & $\begin{array}{c}\text { Trissomia } 18 \\
(\mathrm{n}=26)\end{array}$ & $\begin{array}{c}\text { Trissomia } 21 \\
(n=25)\end{array}$ & $\begin{array}{c}\text { Valor } \\
p\end{array}$ \\
\hline Idade materna em anos (média_DP) & $30,4 \pm 8,2$ & $33,1 \pm 8,3$ & $37 \pm 5,9$ & 0,05 \\
\hline Paridade & & & & 0,1 \\
\hline Primípara & 11,1 & 42,3 & 20 & \\
\hline Multípara & 88,9 & 57,7 & 80 & \\
\hline Idade gestacional & & & & 0,6 \\
\hline Segundo trimestre & 44,4 & 69,2 & 68 & \\
\hline Terceiro trimestre & 55,6 & 30,8 & 32 & \\
\hline Sexo fetal & & & & 0,67 \\
\hline Masculino & 77,8 & 57,7 & 60 & \\
\hline Feminino & 22,2 & 42,3 & 40 & \\
\hline Motivo de encaminhamento & & & & $<0,01$ \\
\hline TN alterada & 0 & 11,5 & 44 & \\
\hline MF detectadas & 100 & 73,1 & 40 & \\
\hline Risco materno & 0 & 15,4 & 16 & \\
\hline Desfecho da gestação & & & & 0,9 \\
\hline Óbito fetal intraútero & 55,6 & 50 & 36 & \\
\hline Nativivo & 44,4 & 50 & 64 & \\
\hline
\end{tabular}

TN: translucência nucal; MF: malformações; DP: desvio padrão; p: significância estatística obtida pelos testes de ANOVA (comparação de médias) e exato de Fischer (variáveis categóricas)
Quanto à paridade, a maioria das pacientes já havia tido alguma gestação anteriormente, não havendo diferença entre os grupos.

O diagnóstico de trissomia foi fornecido pela cariotipagem fetal no segundo trimestre, na grande maioria dos casos, nos grupos de trissomia dos cromossomos 18 e 21 , e no terceiro trimestre no grupo de trissomia do cromossomo 13. Nos três grupos, houve mais fetos do sexo masculino, principalmente na trissomia do cromossomo $13(77,8 \%)$.

Com relação ao motivo de encaminhamento para o Serviço de Medicina Fetal do HCPA, todos os casos de trissomia do cromossomo 13 foram referidos devido à detecção de malformações fetais em ultrassonografia obstétrica. Já no grupo de trissomia do cromossomo 18, a maioria $(73,1 \%)$ dos fetos também foi encaminhada por malformações detectadas, porém $15,4 \%$ dos casos apresentavam risco materno aumentado para cromossomopatias e $11,5 \%$ foram encaminhados por TN aumentada. Em relação aos casos de trissomia do cromossomo 21, o principal motivo de encaminhamento foi o aumento da TN (44,0\%), seguido pelo achado de malformações fetais $(40,0 \%)$ e pelo risco materno aumentado para cromossomopatias.

O desfecho gestacional foi relativamente semelhante nos grupos de trissomia dos cromossomos 13 e 18 . Dentre os fetos com trissomia do cromossomo 21, ocorreu óbito fetal em 36\% dos casos identificados.

$\mathrm{Na}$ Tabela 2 estão descritas as principais malformações fetais identificadas nos três grupos de trissomias.

Tabela 2. Principais malformacões congênitas fetais encontradas nas trissomias dos cromossomos 13,18 e 21

\begin{tabular}{|c|c|c|c|c|c|c|c|}
\hline \multirow[t]{2}{*}{ Malformações detectadas } & \multicolumn{2}{|c|}{$\begin{array}{c}\text { Trissomia } \\
13\end{array}$} & \multicolumn{2}{|c|}{$\begin{array}{c}\text { Trissomia } \\
18\end{array}$} & \multicolumn{2}{|c|}{$\begin{array}{c}\text { Trissomia } \\
21\end{array}$} & \multirow{2}{*}{$\begin{array}{c}\text { Valor } \\
\mathbf{p}\end{array}$} \\
\hline & $n$ & $\%$ & $\mathrm{n}$ & $\%$ & $n$ & $\%$ & \\
\hline Comunicação interatrial & 1 & 11,1 & 3 & 11,5 & 5 & 20,0 & 0,79 \\
\hline Comunicação interventricular & 6 & 66,7 & 9 & 34,6 & 6 & 24,0 & 0,09 \\
\hline Defeito do septo átrio-ventricular & 0 & 0 & 4 & 15,4 & 5 & 20,0 & 0,49 \\
\hline Hipoplasia do ventrículo esquerdo & 2 & 22,2 & 3 & 11,5 & 1 & 4,0 & 0,24 \\
\hline Onfalocele & 2 & 22,2 & 10 & 38,5 & 0 & 0 & $<0,01$ \\
\hline Pielectasia & 5 & 55,6 & 3 & 11,5 & 0 & 0 & $<0,01$ \\
\hline Genitália ambígua & 3 & 33,3 & 2 & 7,7 & 0 & 0 & 0,01 \\
\hline Hérnia diafragmática & 0 & 0 & 3 & 11,5 & 0 & 0 & 0,20 \\
\hline Ventriculomegalia & 4 & 44,4 & 5 & 19,2 & 5 & 20,0 & 0,31 \\
\hline Holoprosencefalia & 4 & 44,4 & 0 & 0 & 0 & 0 & $<0,01$ \\
\hline Meningomielocele & 1 & 11,1 & 5 & 19,2 & 0 & 0 & 0,06 \\
\hline Encefalocele & 2 & 22,2 & 1 & 3,8 & 0 & 0 & 0,06 \\
\hline Fenda labial/palatina & 6 & 66,7 & 1 & 3,8 & 0 & 0 & $<0,01$ \\
\hline Encurtamento de ossos longos & 0 & 0 & 2 & 7,7 & 5 & 20,0 & 0,29 \\
\hline Pé torto congênito & 2 & 22,2 & 12 & 46,2 & 1 & 4,0 & $<0,01$ \\
\hline Defeitos nas mãos & 4 & 44,4 & 13 & 50,0 & 2 & 8,0 & $<0,01$ \\
\hline Artéria umbilical única & 0 & 0 & 2 & 7,7 & 0 & 0 & 0,15 \\
\hline
\end{tabular}


As cardiopatias pesquisadas ocorreram, na maioria dos casos, nos três grupos (29,8\% do total de malformações), sendo a comunicação interventricular (CIV) a principal cardiopatia identificada, especialmente no grupo da trissomia do cromossomo $13(66,7 \%)$.

Dentre os casos de malformações gastrintestinais, a onfalocele foi significativamente mais frequente nos casos de trissomia do cromossomo $18(\mathrm{p}<0,01)$.

As anomalias geniturinárias ocorreram, principalmente, na trissomia do cromossomo 13 , porém mais da metade dos casos desta trissomia apresentou pielectasia $(55,6 \%)$ significativamente maior neste grupo $(\mathrm{p}<0,01)$ e o mesmo ocorreu com a genitália ambígua $(33,3 \% ; \mathrm{p}=0,01)$.

O achado de hérnia diafragmática, representante das malformações respiratórias, apareceu somente no grupo de trissomia do cromossomo 18 , em três casos $(11,5 \%)$.

Defeitos do SNC foram identificados em todos os casos de trissomia do cromossomo 13, que é o grupo responsável por todos os casos de holoprosencefalia. A ventriculomegalia foi demonstrada em quatro casos de trissomia $13(44,4 \%)$, em cinco de trissomia 18 (19,2\%), e em cinco de trissomia $21(20,0 \%)$. As fendas faciais (labiais e/ou palatinas) foram significativamente mais prevalentes nos fetos com trissomia do cromossomo 13 (66,7\%; $\mathrm{p}<0,01)$.

Dentre os defeitos dos membros, cinco fetos $(20 \%)$ com trissomia do cromossomo 21 apresentaram encurtamento de ossos longos, o qual foi o achado mais comum neste grupo. Em relação às malformações nas mãos e nos pés, houve diferença estatisticamente significativa entre os grupos de trissomia: os defeitos nas mãos ocorreram em 13 casos de trissomia do cromossomo $18(50 \%$; $\mathrm{p}<0,01)$ e em quatro casos de trissomia do cromossomo $13(44,4 \%)$; já o achado de pé torto congênito foi mais comum no grupo de trissomia do cromossomo 18 , sendo descrito em 12 fetos $(46,2 \% ; \mathrm{p}<0,01)$. Os casos de artéria umbilical única ocorreram no grupo de trissomia 18 .

Foram descritas ao todo 161 malformações, em 50,9; 27,3 e 21,7\% dos casos de trissomias 18, 13 e 21 , respectivamente. Como achado ultrassonográfico relevante, dois casos de trissomia 13 (22,2\%), 12 casos de trissomia $18(46,1 \%)$ e sete de trissomia $21(28 \%)$ apresentaram polidramnia durante a gestação. Da mesma forma, a hidropisia foi identificada em um caso $(11,1 \%)$ de trissomia 13 , dois $(7,7 \%)$ de trissomia 18 e dois $(8 \%)$ de trissomia 21.

\section{Discussão}

Existem inúmeras malformações descritas associadas às trissomias, além das próprias características fenotípicas de cada síndrome. Numa revisão incluindo 33 casos de trissomia do cromossomo 13, 91\% dos fetos apresentavam uma ou mais anormalidades no exame ultrassonográfico pré-natal. As principais malformações descritas foram: holoprosencefalia (39\%), outras anormalidades do SNC $(58 \%)$, anormalidades faciais $(48 \%)$, renais $(33 \%)$ e cardíacas $(48 \%)^{9}$. Da mesma forma, em outro estudo, demonstrou-se que fetos com trissomia do cromossomo 13 apresentavam frequentemente defeitos cardíacos, anomalias faciais e do $\mathrm{SNC}^{10}$. A holoprosencefalia encontra-se associada à onfalocele em aproximadamente $40 \%$ dos casos de trissomia 13, e as anomalias faciais também a acompanham, principalmente os defeitos de linha média, como hipotelorismo, fendas labiopalatinas, narina única, probóscide e ciclopia ${ }^{11,12}$. Dentre as malformações renais, os rins displásicos acometem $48 \%$ dos $\operatorname{casos}^{11}$. Em um estudo recente, as malformações mais comuns nos fetos com trissomia 13 foram os defeitos craniofaciais, as malformações cerebrais e as anomalias do trato geniturinário ${ }^{13}$. Neste trabalho, as principais malformações identificadas nos fetos com trissomia do cromossomo 13 foram as fendas faciais e a CIV, ambas identificadas em 66,7\% dos casos; as alterações geniturinárias representadas pela pielectasia em 55,6\% e pela genitália ambígua em 33,3\%. Dentre as anomalias do SNC, $44,4 \%$ dos fetos apresentaram holoprosencefalia e ventriculomegalia. A onfalocele foi encontrada em 22,2\% dos casos.

Com relação à trissomia do cromossomo 18 , há descrição na literatura sobre mais de 130 anomalias diferentes e nenhuma foi considerada patognomônica da síndrome ${ }^{14,15}$. Em um estudo, foram descritos 70 casos de trissomia 18, no qual 61 fetos $(87,1 \%)$ apresentavam uma ou duas anomalias maiores e as cardiopatias foram as mais encontradas $(47,1 \%)$, com incidência de $27,1 \%$ dos defeitos de septo ventricular. As malformações do SNC foram identificadas em $35,7 \%$ dos casos, as anormalidades faciais em $14,3 \%$ dos casos, os defeitos gastrintestinais em $10 \%$ e geniturinários em $4,3 \%^{16}$. Em outro estudo, relatou-se que $80 \%$ dos fetos com trissomia 18 apresentaram malformações cardíacas estruturais ${ }^{17}$. Holmgren e Lacoursiere ${ }^{6}$ avaliaram 20 casos de trissomia do cromossomo 18 e encontraram defeitos de parede abdominal em $25 \%$, malformações do SNC em $65 \%$ e cardiopatias em $95 \%$, principalmente defeitos septais ventriculares e patência de ducto arterioso. Em outro estudo que incluiu 98 fetos com trissomia do cromossomo 18, os achados ultrassonográficos anormais mais frequentemente identificados foram os defeitos cardíacos e do SNC, presentes em 63 e $34 \%$ dos casos, respectivamente ${ }^{18}$. Dentre as anomalias do sistema gastrintestinal, está descrito que as mais usualmente encontradas em fetos com trissomia do cromossomo 18 são: divertículo de Meckel, onfalocele e má rotação intestinal ${ }^{11}$.

As malformações das extremidades inferiores e dos pés já foram encontradas em quase dois terços dos fetos com trissomia $18^{19}$. No geral, as malformações mais 
comumente descritas em fetos com trissomia do cromossomo 18 envolvem cérebro, coração e membros ${ }^{20}$. Em decorrência destas malformações, é bastante comum o achado de crescimento intrauterino restrito ${ }^{13}$. No presente trabalho, as principais malformações identificadas nos fetos com trissomia do cromossomo 18 foram os defeitos nas mãos $(50,0 \%)$, seguidos de pés tortos congênitos $(46,2 \%)$ e onfalocele $(38,5 \%)$, com consequente polidramnia. Dentre as cardiopatias, o principal achado neste grupo foi a CIV (34,9\%). Os defeitos do SNC foram observados em apenas $19,2 \%$ dos casos, caracterizados como ventriculomegalia. Do total de malformações apresentadas no estudo, 50,9\% delas foram neste grupo. A avaliação da restrição de crescimento não foi o objetivo deste estudo.

Aproximadamente um terço dos fetos com trissomia do cromossomo 21 apresentam uma ou mais malformações estruturais ultrassonograficamente detectáveis ${ }^{21}$. Dentre as descritas, sabe-se que fetos com trissomia do cromossomo 21 mostram elevada incidência de malformações cardíacas, podendo chegar a $65 \%$ em alguns estudos, principalmente defeitos septais ${ }^{11}$. Entre os casos de trissomia do cromossomo 21 , o achado ultrassonográfico mais comum foi a cardiopatia ${ }^{10}$. Outras malformações fortemente associadas à trissomia do cromossomo 21 são: hidrocefalia, hipoplasia cerebelar, atresia de duodeno, onfalocele e anomalias de membros $^{11}$. Aproximadamente 17 a $25 \%$ dos fetos com trissomia do cromossomo 21 apresentam hidronefrose descoberta durante o pré-natal, em comparação com 2 a $3 \%$ dos fetos com cariótipo normal ${ }^{6}$. Num estudo de coorte retrospectivo de 1.055 gestações com achado isolado de pielectasia fetal, confirmou-se a associação entre este achado e o aumento de risco das aneuploidias, especialmente de trissomia do cromossomo 21 (aumento de pelo menos duas vezes o risco) $)^{22}$.

Um estudo recente concluiu que a detecção isolada de pielectasia fetal no segundo trimestre está associada com um aumento da possibilidade diagnóstica de trissomia $21^{23}$. Em 2014, publicou-se um estudo de revisão sistemática sobre as regras de predição clínica para o cálculo do risco de síndrome de Down, baseadas em achados ultrassonográficos no segundo trimestre de gestação. Este artigo, após revisar 10 preditores clínicos, sendo dois com validação interna e um com externa, concluiu que os resultados não são concordantes e podem gerar situações de superestimação ou subestimação do risco de trissomia do cromossomo 21, apesar das inúmeras evidências sobre o fenótipo dos fetos esta trissomia ${ }^{24}$. Dentre as malformações presentes nos casos de trissomia do cromossomo 21 identificados neste trabalho, destacam-se as cardiopatias, principalmente CIV (24,0\%), comunicação interatrial $(20,0 \%)$ e defeito de septo atrioventricular - DSAV (20,0\%). Além disso, tais fetos apresentaram ventriculomegalia $(20,0 \%)$ e encurtamento de ossos longos (20,0\%).
A taxa para detecção de anomalias fetais relacionadas diretamente ao diagnóstico de cromossomopatias depende essencialmente da qualificação e experiência dos ultrassonografistas, da qualidade dos ecógrafos e do nível e duração dos exames ultrassonográficos obstétricos ${ }^{10}$. Corroborando esta ideia, em estudo sobre o diagnóstico pré-natal de anormalidades cromossômicas, por meio de testes invasivos, verificou-se que a maioria dos casos de alterações em cariótipos fetais foi encontrada no grupo encaminhado por malformações identificadas em ultrassonografias ${ }^{25}$. Além disso, o cariótipo permanece como uma ferramenta fundamental no momento de avaliar pacientes com malformações cardíacas e de qualquer índole, tendo presente que a necessidade de identificar as malformações fetais e possibilitar a cariotipagem antenatal cresce na medida em que, nas patologias letais, o risco de óbito intrauterino é alto e a autólise muitas vezes torna a necropsia impossível. Isso dificulta o diagnóstico etiológico e prejudica o aconselhamento dos pais sobre os riscos fetais nas futuras gestações ${ }^{8,26}$.

Em estudo de necropsias fetais, Tennstedt et al. descreveram que, dentre os 129 fetos que apresentavam cardiopatias, anormalidades cromossômicas foram diagnosticadas em 43 casos (33\%), sendo que 19, 11 e 6 fetos apresentavam trissomias do 21, 18 e 13, respectivamente. Também foi apresentado que 30 e $12 \%$ dos casos de DSAV identificados foram associados à trissomia dos cromossomos 21 e 18 , respectivamente ${ }^{27}$. As cardiopatias foram as malformações mais encontradas nos três grupos do presente estudo (29,8\%). O complexo de Dandy-Walker (ventriculomegalia, alargamento da cisterna magna e defeito do vermix cerebelar com dilatação cística do quarto ventrículo) ${ }^{28}$ é considerado um marcador de trissomia do cromossomo 18, porém também ocorre na trissomia do cromossomo 13 . Em relação a outras malformações do SNC, a holoprosencefalia e a meningocele são mais comuns na trissomia do cromossomo $13 \mathrm{e}$ a ventriculomegalia, na trissomia do cromossomo $18^{11}$.

Com o avanço da idade materna, tem-se um risco fetal aumentado de aneuploidias. Devido a estes fetos terem um risco maior de óbito intrauterino, quanto maior for a idade gestacional menor será o risco de anomalia cromossômica ${ }^{29}$. Assim, para aconselhamento genético pré-natal e pré-concepcional, o estudo da etiologia das malformações associadas às síndromes por meio do cariótipo fetal mostra-se uma ferramenta importante. Considerando a incidência crescente de aneuploidias, em virtude da opção atual das mulheres por gestação mais tardia, tornam-se necessários tais estudos com a finalidade de descrever os achados sugestivos, as diferenças de desfechos encontradas e as possibilidades de diagnóstico fetal com riscos minimizados. 
1. Evans MI, Ebrahim SA, Berry SM, Holzgreve W, Isada NB, Quintero RA, et al. Fluorescent in situ hybridization utilization for high-risk prenatal diagnosis: a trade-off among speed, expense, and inherent limitations of chromosome-specific probes. Am J Obstet Gynecol. 1994;171(4):1055-7.

2. Hook EB, Cross PK, Schreinemachers DM. Chromosomal abnormality rates at amniocentesis and in live-born infants. JAMA. 1983;249(15):2034-8.

3. Nicolaides $\mathrm{KH}$. Screening for chromosomal defects. Ultrasound Obstet Gynecol. 2003;21 (4):313-21.

4. Milner AD, Hoskyns EW, Hopkin IE. The effects of mid-trimester amniocentesis on lung function in the neonatal period. Eur J Pediatr. $1992 ; 151(6): 458-60$.

5. Drugan A, Johnson MP, Evans MI. Ultrasound screening for fetal chromosome anomalies. Am J Med Genet. 2000;90(2):98-107.

6. Holmgren C, Lacoursiere DY. The use of prenatal ultrasound for the detection of fetal aneuploidy. Clin Obstet Gynecol. 2008;51 (1):48-61.

7. Nicolaides KH, Snijders RJ, Gosden CM, Berry C, Campbell S. Ultrasonographically detectable markers of fetal chromosomal abnormalities. Lancet. 1992;340(8821):704-7.

8. Agathokleous M, Chaveeva P, Poon LC, Kosinski P, Nicolaides $\mathrm{KH}$. Meta-analysis of second-trimester markers for trisomy 21. Ultrasound Obstet Gynecol. 2013;41(3):247-61.

9. Lehman CD, Nyberg DA, Winter TC 3rd, Kapur RP, Resta RG, Luthy DA. Trisomy 13 syndrome: prenatal US findings in a review of 33 cases. Radiology. 1995;194(1):217-22.

10. Papp C, Szigeti Z, Tóth-Pál E, Hadjú J, Joó JG, Papp Z. Ultrasonographic findings of fetal aneuploidies in the second trimester - our experiences. Fetal Diagn Ther. 2008;23(2):105-13.

11. Pastore AR. Ultrassonografia em ginecologia e obstetrícia. 3 ed. Rio de Janeiro: Revinter; 2010.

12. Melo NR, Fonseca EB. Medicina fetal. Rio de Janeiro: Elsevier; 2012.

13. Kroes I, Janssens S, Defoort P. Ultrasound features in trisomy 13 (Patau syndrome) and trisomy 18 (Edwards syndrome) in a consecutive series of 47 cases. Facts Views Vis Obgyn. 2014;6(4):245-9.

14. Rosa RF, Rosa RC, Zen PR, Graziadio C, Paskulin GA. Trissomia 18: revisão dos aspectos clínicos, etiológicos, prognósticos e éticos. Rev Paul Pediatr. 2013;31(1):111-20.

15. Denardin D, Savaris FE, Cunha AC, Betat RD, Telles JA, Targa LV, et al. Retrospective cohort of trisomy 18 (Edwards syndrome) in southern Brazil. Sao Paulo Med J. 2014 Nov 7. [Epub ahead of print]

16. Papp C, Ban Z, Szigeti Z, Csaba A, Beke A, Papp Z. Role of second trimester sonography in detecting trisomy 18: a review of 70 cases. J Clin Ultrasound. 2007;35(2):68-72.
17. DeVore GR. Second trimester ultrasonography may identify 77 to $97 \%$ of fetuses with trisomy 18. J Ultrasound Med. 2000; 19(8):565-76.

18. Watson WJ, Miller RC, Wax JR, Hansen WF, Yamamura Y, Polzin WJ. Sonographic findings of trisomy 18 in the second trimester of pregnancy. J Ultrasound Med. 2008;27(7):1033-8.

19. Yeo L, Guzman ER, Day-Salvatore D, Walters C, Chavez D, Vintzileos AM. Prenatal detection of fetal trisomy 18 through abnormal sonographic features. J Ultrasound Med. 2003;22(6):581-90.

20. Bronsteen $\mathrm{R}$, Lee $\mathrm{W}$, Vettraino IM, Huang $\mathrm{R}$, Comstock $\mathrm{CH}$. Second-trimester sonography and trisomy 18. J Ultrasound Med. 2004;23(2):233-40.

21. Spicer RL. Cardiovascular disease in Down syndrome. Pediatr Clin North Am. 1984;31(6):1331-43.

22. Carbone JF, Tuuli MG, Dicke JM, Macones GA, Odibo AO. Revisiting the risk for aneuploidy in fetuses with isolated pyelectasis. Prenat Diagn. 2011;31(6):566-70.

23. Orzechowski KM, Berghella V. Isolated fetal pyelectasis and the risk of Down syndrome: a meta-analysis. Ultrasound Obstet Gynecol. 2013;42(6):615-21.

24. Moreno-Cid M, Tenías Burillo JM, Rubio-Lorente A, Rodríguez M, Bueno-Pacheco G, Román-Ortiz C, et al. Systematic review of the clinical prediction rules for the calculation of the risk of Down syndrome based on ultrasound findings in the second trimester of pregnancy. Prenat Diagn. 2014;34(3):265-72.

25. Kessler RG, Sanseverino MT, Leistner-Segal S, Magalhães JA, Giugliani R. Prenatal diagnosis of fetal chromosomal abnormalities: report of an 18-year experience in a Brazilian public hospital. Genet Mol Biol. 2008;31 (4):829-33.

26. Trevisan P, Rosa RF, Koshiyama DB, Zen TD, Paskulin GA, Zen PR. Congenital heart disease and chromossomopathies detected by the karyotype. Rev Paul Pediatr. 2014;32(2):262-71.

27. Tennstedt C, Chaoui R, Körner H, Dietel M. Spectrum of congenital heart defects and extracardiac malformations associated with chromosomal abnormalities: results of a seven year necropsy study. Heart. 1999;82(1):34-9.

28. De Patta CB, Galluzzo RN, Da Correggio KS, Trapani A Jr, Corrêa AM, Travesso DJ. Malformação de Dandy-Walker: diagnóstico pré-natal: relato de caso. ACM Arq Catarin Med. 2013;42(3):71-5.

29. Nicolaides KH, Duarte LB, Marcolim AC, Duarte G. [First-trimester screening for chromosomal abnormalities]. Rev Bras Ginecol Obstet. 2007;29(12):647-53. Portuguese. 